\title{
Respiratory Care Year in Review 2013: Airway Management, Noninvasive Monitoring, and Invasive Mechanical Ventilation
}

\author{
Charles G Durbin Jr MD FAARC, Lluís Blanch MD, Eddy Fan MD PhD, \\ and Dean R Hess PhD RRT FAARC
}

\author{
Introduction \\ Airway Management \\ Emergency Airway Management \\ Management of the Difficult Airway \\ Airway Tube Maintenance Issues \\ Noninvasive Respiratory Monitoring \\ Patient Observation \\ Gas Exchange and Alveolar Ventilation \\ Respiratory Mechanics, Lung Volume, and Lung Densities Distribution \\ Central Drive and Respiratory Muscles \\ Information Technologies \\ Invasive Mechanical Ventilation \\ High-Frequency Oscillatory Ventilation in Adult Patients With ARDS \\ Lung-Protective Ventilation Outside the ICU \\ Tracheostomy Timing and Weaning \\ Summary
}

Fundamental to respiratory care practice are airway management, noninvasive monitoring, and invasive mechanical ventilation. The purpose of this paper is to review the recent literature related to these topics in a manner that is most likely to have interest to the readers of Respiratory Care. Key words: extubation; high-frequency oscillatory ventilation; intubation; lung-protective ventilation; mechanical ventilation; respiratory mechanics; tracheostomy. [Respir Care 2014;59(4):595-606. (C) 2014 Daedalus Enterprises]

\section{Introduction}

It remains a challenge to review the literature relevant to one's practice and then update that practice based on the current evidence. At the American Association for Respiratory Care Congress 2013, RESPIRATORY CARE presented a series of lectures on the theme of "Year in Review." Topics were chosen that are likely to have special interest to the readers of the Journal. In this paper, we publish topics

\footnotetext{
Care Department, Massachusetts General Hospital, and the Department of Anesthesia, Harvard Medical School, Boston, Massachusetts.

A version of this paper was presented by Drs. Durbin, Blanch, and Fan at the 59th International Respiratory Convention \& Exhibition of the American Association for Respiratory Care Congress 2013, held November 16-19, 2013, in Anaheim, California.
} 
related to airway management, noninvasive monitoring, and invasive mechanical ventilation.

\section{Airway Management}

2013 has been a bumper year for interesting and important publications related to airway management. Updated and comprehensive algorithms for dealing with difficulties occurring during intubation, how to recognize and prepare for risky extubations, when to perform a tracheostomy or other surgical airway, and methods to approach cardiac and airway emergencies have been published.

\section{Emergency Airway Management}

One of the difficulties in managing emergencies is the unpredictability of when these events occur. Having appropriate equipment and qualified staff rapidly available can reduce the adverse impact of emergency intubations. A paper by Adams et $\mathrm{al}^{1}$ analyzed almost 1,500 urgent or emergent intubations over a 3-year period. They classified these events by the time of day and month of the year. The motivation behind this report is to improve the outcomes of these rare events by identifying the peak times they occur and matching resources. This retrospective report included all consultations for the airway team that resulted in an intubation at a major urban academic medical center. Contrary to common beliefs, the principal finding of the study is that the majority of urgent and emergent intubations (57\%) took place during the daytime hours of 7 AM to 7 PM. Not unexpectedly, a majority of airway consultations for intubation occurred during the winter and early spring months, which is the peak incidence of community-acquired pneumonia.

The incidence and time distribution of difficult intubations were seen in $11 \%$ of cases and were equally distributed between day and night shifts. A significant limitation of this paper is that it included only a few of the intubation events occurring in the emergency department, as the consult airway team was rarely asked for assistance in this setting. In studying the daily variation of intubation frequency, the peak time for intubation was 3 PM to 7 PM, and

Dr Durbin discloses relationships with Masimo Corporation and Kimberly-Clark Corporation. Dr Blanch discloses a relationship with Corporació Sanitària Parc Taulí. Dr Fan has declared no conflicts of interest. Dr Hess discloses relationships with Philips Respironics, Pari Respiratory Equipment, Covidien, Maquet, Merck, and Bayer Corporation.

Correspondence: Dean Hess PhD RRT FAARC, Respiratory Care, Massachusetts General Hospital, 55 Fruit Street, Boston, MA 02114. E-mail: dhess@partners.org.

DOI: $10.4187 /$ respcare.03199 the nadir was 11 PM to 3 AM. Overall, these data may help managers in other institutions determine how to schedule staff for these unpredictable events. The airway team in this 900 -bed institution is available $24 \mathrm{~h}$ a day and consists of an ICU anesthesia resident, a respiratory therapist, a critical care fellow, and an ICU faculty attending. This degree of expertise is generally not available around the clock in many hospitals, so being aware of the most frequent times of need may help managers to plan for these events.

Airway competence and skills of newly graduated intensivists were the subject of a national survey published last year in Respiratory CARE. ${ }^{2}$ Critical care and pulmonary critical care fellowship programs $(n=168)$ were asked about their airway and intubation education and experience. Of the $56 \%$ of programs that responded, fellows received widely varied experiences in the number of intubations, the use of devices, and the quality of airway experience. Most programs failed to achieve the minimum number of procedures the program directors felt were necessary for developing competence with many of the airway management techniques that are now available. While this survey suggests widespread deficits in airway education and experience, competence is a more important outcome than simply the number of procedures performed. Determination of individual success and competence would be a better marker of the quality of the training programs. With an expanding armamentarium of airway devices, achieving and maintaining adequate skills using all of them will be difficult in both residency and clinical practice. Starting with mastery of direct laryngoscopy, some educators are now recommending that one or at most two additional techniques for tracheal intubation be mastered by all critical care fellows. How to develop and demonstrate this level of competence needs to be defined.

Airway management during cardiopulmonary resuscitation (CPR) has been the subject of discussion and study for the past few years. New recommendations for layperson CPR emphasize aggressive chest compression only without ventilation until advanced life support or a defibrillator arrives. ${ }^{3}$ Coronary perfusion pressure is a critical predictor of eventual resuscitation success, and any interruption or slowing of compressions results in rapid and sustained fall in this pressure. Hospital and emergency first responders are now encouraged to follow a similar basic approach by adding 2 ventilations for every 30 compressions, with no interruption of compressions. Intubation is delayed until defibrillation is attempted or determined not to be appropriate.

The effect of airway management on the development of pneumonia after successful cardiac resuscitation is of interest. In a previous study, Pabst et $\mathrm{al}^{4}$ hypothesized that replacing the field-placed endotracheal tube (ETT) on arrival at the hospital would decrease the incidence of early- 
onset pneumonia in comatose patients following cardiac resuscitation in the community. All subjects underwent therapeutic hypothermia $\left(32-34^{\circ} \mathrm{C}\right)$ for $24 \mathrm{~h}$ by external cooling with ice packs and a cooling mat, as well as by internal cooling with cold infusions. ETT changes were performed at random, assuming unsterile ETT handling during out-of-hospital CPR. The ETT was also exchanged if the field-inserted ETT was too small for the subject's body size. Patients with supraglottic airway devices had them replaced by an ETT immediately after hospital admission. Patients were excluded if they died within the first $24 \mathrm{~h}$ after hospital admission. In the 104 patients entered into the study, the rate of early-onset pneumonia $(50 \%)$ was not significantly changed by exchanging the ETT that had been inserted during resuscitation. However, early-onset pneumonia was associated with a longer period of mechanical ventilation and ICU stay but did not contribute to increased mortality. The ICU mortality was $22.8 \%$ and was not different between the two ETT management groups. Patients with observed aspiration (30\% of the total) fared no worse than those without confirmed aspiration. A conclusion from this study is that changing the ETT even with known aspiration at the scene to prevent early-onset pneumonia is not an effective preventive measure.

A laryngeal tube airway 5 was used in the field and changed to an ETT in 6 of the subjects in the above report. Occasionally, the laryngeal tube airway is inserted due to prolonged and failed intubation attempts. Tongue and pharyngeal swelling can occur and may make removal of this device even more challenging and potentially lethal. Some have recommended that a surgical airway be placed if the device is successful and signs of airway swelling or edema, such as a swollen protruding tongue, are present. A paper this year by Khaja and Chang ${ }^{6}$ reported on 3 patients with these findings who received management directed by fiberoptic scope (FOS) examination with the airway device in place. All patients had a stable airway on arrival, and ventilation was adequate with the device. All were taken to the operating room for management. Only one patient received a tracheostomy; the other two were able to be safely intubated using the flexible scope and a variety of other airway devices.

Airway management attempts and the placement of the King LT device can result in significant soft tissue swelling of the airway. The tongue of the third patient in this series was noted to be protuberant, similar to the presentation of patients in other series requiring tracheostomy. However, examination with the FOS and GlideScope video laryngoscope demonstrated that the swelling was limited primarily to the anterior tongue, and in fact, within minutes of deflating the cuff and removing the King LT, the swelling significantly improved. This lends support to the idea that the swelling is often secondary to inflation of the cuff on the King LT airway itself. Gaither et $\mathrm{al}^{7}$ hypothesized that the lingual engorgement was secondary to compromise of venous drainage from the tongue. With the use of flexible fiberoptic laryngoscopy, a technique widely utilized in most institutions, it was possible to determine the location of the King LT in the airway and thereby to allow decisions to be made about definitive airway management. The authors suggest an algorithm utilizing the FOS to help decide how to manage the King LT pharyngeal tube airway removal when signs of swelling are present.

There have been a plethora of new and modified airway management devices introduced into clinical practice over the past 20 years. A recent review of this expanding domain was published this year by Ostermayer and GauscheHill. ${ }^{8}$ They present a brief history of the development of many of the new devices, as well as a summary of the published data supporting each device's use. Of note, they include pediatric and neonatal devices, as well as those for adult patients, and a useful table listing specifics about the individual devices, associated benefits, and reported complications.

Complications related to emergency airway management go beyond failure to provide adequate ventilation and oxygenation. Airway manipulation and tracheal intubation elicit sympathetic responses that can cause hypertension and tachycardia, conditions exacerbating the potential for cardiac ischemia, excessive bleeding, or stroke. Following a rise in blood pressure is a compensatory response by the parasympathetic system leading to bradycardia and possibly asystolic cardiac arrest. Once intubated, positive-pressure ventilation reduces venous return and aggravates hypotension and shock. These changes in blood pressure are commonly seen during elective intubations in the operating room, and proactive measures are used to minimize the deleterious effects they may cause. Unfortunately, these hemodynamic changes also occur during emergency intubations.

Hasegawa et $\mathrm{al}^{9}$ report on a large multi-center prospective study of patients during emergency intubation in the emergency ward in 13 Japanese hospitals. Of the nearly 2,000 patients reported, most of whom were intubated for medical reasons $(>80 \%), 2 \%$ sustained significant hypotension following intubation (systolic blood pressure $<90 \mathrm{~mm} \mathrm{Hg}$ ). For the patients over 65 years of age, this incidence was 3 times higher. No cardiac arrests related to intubation were reported in this study. A multivariate analysis of all patients demonstrated that age 65 years or older was an independent predictor of post-intubation hypotension.

In a less optimistic report from the United States, Heffner et $\mathrm{al}^{10}$ reported on cardiac arrests following emergency room intubations in a single institution. Unlike the previous report with no cardiac arrests, the incidence of 
post-intubation cardiac arrest was $4.2 \%$ in this group of 410 adult patients. Differences in intubation techniques or patient characteristics might account for some of this difference. Most intubations in this study were performed using a rapid sequence intubation technique with sedation to unconsciousness and a muscle relaxant. The Japanese paper employed this technique in only $20 \%$ of their intubations. Resuscitation was attempted in all patients suffering post-intubation cardiac arrest but was successful in just over half of them. One-hundred eight of the 410 patients died in the hospital, an overall mortality rate of $26 \%$. Patients experiencing cardiac arrest had higher in-hospital mortality (82 vs $24 \%, P<.001$ ). Peri-intubation cardiac arrest was associated with a 14-fold increase in odds of hospital death (odds ratio 14.8, 95\% CI 4.2-52\%) compared to patients without cardiac arrest related to intubation. All intubations, but especially emergency intubations, create opportunities for severe hemodynamic alterations that can affect survival. Anticipation and treatment of these perturbations should be part of all airway management programs.

\section{Management of the Difficult Airway}

There were two important papers on difficult airway management published early this year authored by the Canadian Airway Focus Group. ${ }^{11,12}$ These papers review the published literature and update recommendations published by the group in 1998. The data evaluation and recommendation system has been improved and uses the Grading of Recommendations Assessment, Development, and Evaluation (GRADE) system. This system includes presenting the strength of the recommendation, as well as an objective grade for the strength of supporting data. These papers make recommendations for dealing with a difficult airway encountered in the unconscious patient (Part 1) ${ }^{11}$ and for dealing with an anticipated difficult airway (Part 2). ${ }^{12}$ Together, these papers provide guidance for a wide range of problems that are encountered in routine clinical practice both in the operating room and in other areas. There are 19 authors in the group, and the papers include over 20 updated recommendations and more than 400 references. Besides including reviews of a host of new airway devices, the focus of these updates is on prevention of harm and is summarized in the Part 1 conclusion: "The clinician must be aware of the potential for harm to the patient that can occur with multiple attempts at tracheal intubation. This likelihood can be minimized by moving early from an unsuccessful primary intubation technique to an alternative "Plan B" technique if oxygenation by face mask or ventilation using a supraglottic device is non-problematic. Irrespective of the technique(s) used, failure to achieve successful tracheal intubation in a maximum of three attempts defines failed tracheal intubation and signals the need to engage an exit strategy [author emphasis]. Failure to oxygenate by face mask or supraglottic device ventilation occurring in conjunction with failed tracheal intubation defines a failed oxygenation, 'cannot intubate, cannot oxygenate' situation. Cricothyrotomy must then be undertaken without delay, although if not already tried, an expedited and concurrent attempt can be made to place a supraglottic device."

Algorithms incorporating this concern for safety are presented in the papers. Mandating the use of an exit strategy before airway failure leads irretrievably to death is emphasized (ie, no more than 3 attempts at intubation). This is feasible only if the equipment (eg, cricothyrotomy kit) is prepared and ready and the intubator has the appropriate skills. Other areas of important new information include summarizing predictors of when intubation will be difficult, defining "adequate pre-oxygenation (an expired $\mathrm{F}_{\mathrm{IO}_{2}}>90 \%$ )," suggesting strategies for management of extubation of the patient with a difficult airway (ie, leaving a tube-changing device in place or extubating in the operating room with surgeons available), and presenting a suggested form letter to be given to patients after a difficult airway is encountered. These two papers are useful for reading and for reference for both beginners and experienced airway experts.

Cricothyrotomy or other surgical airway, an essential exit strategy in most airway algorithms, is rarely necessary but is mandated to be performed without delay in these guidelines when tracheal intubation and oxygenation fail. How can one acquire skill in performing this procedure? A paper this year suggests that performing at least 5 of these procedures on a model decreases the time to success from skin to lung ventilation to $<60 \mathrm{s.}{ }^{13}$ How this will relate to actual performance is not known. Also, the deterioration in this skill over time has not been evaluated. The act of performing a cricothyrotomy in a dying human is much different than performing the maneuver in a model; however, practice is essential to learning proper use of the equipment. A paper published in 2011 includes details of several different approaches to a percutaneous emergency airway intervention and has excellent illustrations. ${ }^{14}$ This paper is recommended for all clinicians engaged in management of airways.

\section{Airway Tube Maintenance Issues}

Tracheostomy tubes were the subject of several significant papers this year. The long-awaited publication of the TracMan trial appeared in The Journal of the American Medical Association in May. ${ }^{15}$ The data from this large, randomized, prospective study, which has been presented orally at many forums, demonstrated no clinical benefit in having a tracheostomy at day 4 or sooner versus at day 21 . However, 25\% fewer tracheostomies were performed in the 
late group since some patients died and others had been liberated from mechanical ventilation and extubated before day 21. Even the duration of ICU stay was not different between the groups. However, this study was from Great Britain, where stable, intubated patients (with or without a tracheostomy) are generally cared for in high dependence units but not ICUs. These are more similar to a step-down unit in the United States. Most studies in other counties have found that early tracheostomy generates shorter ventilation times and shorter ICU stays, which translates to improved patient comfort and less expense. This large study (904 patients) is reassuring in that there was no increased mortality in the early tracheostomy group and is unlikely to change practice in the United States. This paper does confirm the observation that clinicians are very poor in identifying which patients will need prolonged intubation.

A paper in RESPIRATORY CARE reported shortened length of ICU and hospital stay, quicker use of a speaking valve, and earlier toleration of oral feeding if the initial tracheostomy tube change (downsized) occurred sooner. ${ }^{16}$ The authors prospectively collected data and compared a group of 92 patients who had been divided into those whose initial tracheostomy tube was changed before day 7 and those who had the first tracheostomy tube change after day 7. The multiple services who managed the patients chose tracheostomy change time based on individual service norms, so the study was not randomized. However, mortality was not different between the two groups, suggesting reasonable equivalence. All other studied variables were dramatically shorter in the early change group. There were no complications from tracheostomy tube change in any patient. Besides supporting early downsizing of tracheostomy tubes, having a consistent approach to tracheostomy management, possibly by a dedicated team, could benefit all patients with tracheostomies.

A tracheostomy tube with a lumen opening just above the tube cuff for subglottic secretion removal was shown to be associated with a lower risk of ventilator-associated pneumonia (VAP) than a conventional tube with suction. Ledgerwood et $\mathrm{al}^{17}$ randomized 18 patients to receive the new tube with a subglottic suction port (Portex Blue Line Ultra Suctionaid) or an identical tube from Portex using a traditional open tracheostomy incision and creating a Björk flap. The diagnosis of VAP was more frequent in the group without suction (56 vs 11\%). The study was small, and there was no difference in mortality, although there was a tendency toward longer ventilation and hospital stay in the group with a conventional tube. However, there is a large body of data supporting subglottic suctioning in conventional translaryngeal intubation. Glottic competence is probably impaired by tracheostomy placement and following removal of the translaryngeal intubation, making use of this new tracheostomy tube logically appealing.
Ultrasound is making its way into the airway management arena. Pre-procedure examination of the trachea for overlying vessels or aberrant thyroid tissue is now recommended prior to performing percutaneous dilational tracheostomy. Chou et al ${ }^{18}$ report that correct ETT location in the trachea can be confirmed by ultrasound techniques used during CPR. Six of the 89 studied intubations were esophageal, and 5 of these were correctly recognized by ultrasound. None had $\mathrm{CO}_{2}$ detected with capnography. Five patients had tracheal placement but had no $\mathrm{CO}_{2}$ detected; all were correctly classified with ultrasound, but none had a sustained, effective rhythm at the time, accounting for the negative result by capnography. The use of ultrasonography was helpful in this study of tube placement during cardiac arrest; however, advanced skills and expensive equipment are required. A study using a fiberoptic bronchoscope to confirm correct placement would also demonstrate almost perfect results but would have the same limitations: equipment expense is high, and skills are needed.

Knowing the ETT location in the trachea is critical for avoiding a mainstem bronchial intubation and avoiding accidental extubation. Usually this is done using a plain chest film. The anchoring distance at the lip or teeth is then recorded, and if the chest radiograph shows an undesired tube location, a distance to move the tube is estimated, and this distance is added or subtracted from the marker at the teeth. The consistency of this anchor-to-tip distance has been called into question by a study by Wang et al. ${ }^{19}$ They used repeated chest radiographs after tube repositioning to evaluate how far the tip moved following a change in depth at the teeth. They noted that, when attempting to move the tube a specific distance up or down the trachea, the repeated chest film showed that the tip had moved only about half the desired distance. In fact, in 3 patients, the actual direction of the move was opposite the direction of the tube at the teeth. This discrepancy is due to the mobility of the tube when warmed to body temperature and its ability to arch and bow in the mouth and oral pharynx, leading to no predictable relationship between teeth and tracheal distance.

\section{Noninvasive Respiratory Monitoring}

The status of critically ill patients receiving invasive mechanical ventilation is characterized by rapidly evolving and frequently life-threatening events, which are often difficult to observe by the human eye and might cause important alterations in respiratory system function. Accordingly, it seems highly appropriate to noninvasively monitor respiratory function in patients. In 2013, several papers reported important advances in this field. 


\section{Patient Observation}

Difficult intubation is known to be associated with lifethreatening complications in different scenarios. Although several predictive risk factors and scores for difficult intubation were identified in anesthesia practice, to our knowledge, none have been identified for ICU patients. De Jong et $\mathrm{al}^{20}$ observed that 1,000 difficult intubations from 42 ICUs were strongly associated with moderate and severe life-threatening complications related to intubation. These authors demonstrated that a 7-item simplified score (MACOCHA score) based on patient anatomy, pathology, and operator skills demonstrated good performance in the original group after external validation in a validation cohort (area under the curve $0.89,95 \%$ CI $0.86-0.93$ ). Therefore, a simple score including 7 clinical items discriminates difficult and non-difficult intubation in the ICU.

Huang and $\mathrm{Yu}^{21}$ assessed the predictive value of usual variables for extubation outcome in patients requiring prolonged mechanical ventilation (PMV). After 1 year, 27 of 119 PMV subjects required re-intubation within 7 days, and multivariate logistic regression analysis demonstrated that the only variable associated with extubation failure in patients who tolerated spontaneous breathing trials and were ready to extubate was ineffective cough. The assessment of cough effectiveness at bedside and airway clearance function highlights the importance of airway competence to extubation outcome.

\section{Gas Exchange and Alveolar Ventilation}

The diagnosis of carbon monoxide (CO) poisoning relies on measurement of blood carboxyhemoglobin via venous or arterial blood gas analysis. Due to potential severity and the necessity of immediate therapy, there is an urgent need for rapid and reliable screening to orientate treatment. Two studies have evaluated the performance and accuracy of a lightweight pulse oximeter (Rad-57) enabling noninvasive $\mathrm{CO}$ measurement through a fingertip sensor. Weaver et $\mathrm{al}^{22}$ and Sebbane et $\mathrm{al}^{23}$ in a sample of 1,363 patients and 93 patients, respectively, showed that noninvasive measurement of carboxyhemoglobin with Rad-57 is not sufficiently accurate to direct triage or patient management. Therefore, confirmation with blood $\mathrm{CO}$ measurement by CO-oximetry is still needed. These 2 studies $^{22,23}$ highlighted the importance for respiratory therapists and intensivists to evaluate the cost and efficacy of new technologies. ${ }^{24}$

Siobal et al ${ }^{25}$ calculated mean expired $\mathrm{CO}_{2}\left(\mathrm{P}_{\overline{\mathrm{E} C O}}\right)$ and physiologic dead space (dead-space-to-tidal-volume ratio $\left.\left[\mathrm{V}_{\mathrm{D}} / \mathrm{V}_{\mathrm{T}}\right]\right)$ from the integrated volumetric $\mathrm{CO}_{2}$ monitor in a Dräger XL ventilator and compared them to metabolic analyzer and volumetric $\mathrm{CO}_{2}$ monitor measurements. Measurements in 36 subjects recovering from acute lung injury or ARDS showed that $V_{D} / V_{T}$ was accurately measured by volumetric capnography on the Dräger XL ventilator compared with 3 metabolic analyzers (Metascope, Deltatrac, and Vmax Encore) and the NICO monitor (Philips Respironics). In view of the fact that the measurement of dead space at bedside has important prognostic implications in patients with ARDS, volumetric capnography monitors integrated into ventilators obviate the use of a stand-alone analyzer for measuring $\mathrm{V}_{\mathrm{D}} / \mathrm{V}_{\mathrm{T}}$.

\section{Respiratory Mechanics, Lung Volume, and Lung Densities Distribution}

In patients with ARDS, the selection of ideal PEEP has been extensively investigated, and different methods for selecting the ideal PEEP have been proposed based on respiratory system compliance, oxygenation, and shunt values. Ideal PEEP has also been derived from the analysis of the static inflation and deflation of the pressure-volume curve of the respiratory system. However, after decades of efforts, the results are still controversial. Pintado et $\mathrm{al}^{26}$ studied 70 ARDS patients who were ventilated with low $\mathrm{V}_{\mathrm{T}}$ values and limitation of airway pressure to $30 \mathrm{~cm} \mathrm{H}_{2} \mathrm{O}$ who were randomized to either a compliance-guided PEEP group ${ }^{27}$ or an $\mathrm{F}_{\mathrm{IO}_{2}}$-guided group. ${ }^{28}$ Subjects in the compliance-guided group showed nonsignificant improvements in $\mathrm{P}_{\mathrm{aO}_{2}} / \mathrm{F}_{\mathrm{IO}_{2}}$ during the first 14 days and in 28-day mortality, but multiple-organ-dysfunction-free days, respiratoryfailure-free days, and hemodynamic-failure-free days were significantly lower in subjects with compliance-guided setting of PEEP.

Chiumello et $\mathrm{al}^{29}$ investigated which bedside method would provide PEEP best related to lung recruitability assessed with lung computed tomography scans. To select individual PEEP, bedside methods based on lung mechanics, ${ }^{30,31}$ esophageal pressure, ${ }^{32}$ and oxygenation based on the higher PEEP table of a lung open ventilation (LOV) study ${ }^{33}$ were applied. Most methods provided similar PEEP in mild, moderate, and severe ARDS, unrelated to the lung recruitability. However, as it seems unreasonable to expose lower recruiters (mild ARDS patients) to higher PEEP to keep open a few grams of lung tissue, the LOV method $^{33}$ appears to be the only one that selects a PEEP related to lung recruitability and to the degree of severity of the Berlin Definition, ${ }^{34}$ avoiding higher PEEP in patients with lower recruitability. In fact, Protti et al ${ }^{35}$ studied dynamic and static lung strain by computing the quotient between $\mathrm{V}_{\mathrm{T}}$ and volume of gas caused by PEEP in an experimental pig model with healthy lungs and demonstrated that PEEP protects the lungs by counteracting fluid extravasation and also by limiting tidal ventilation. Large static strains (low $\mathrm{V}_{\mathrm{T}}$ and moderate/high PEEP) are less harmful than large dynamic strains (high $\mathrm{V}_{\mathrm{T}}$ and low PEEP). Therefore, lung 
edema formation depends not only on global strain but also on its components.

Measurement of recruited lung volume and the topographic distribution of $\mathrm{V}_{\mathrm{T}}$ during mechanical ventilation have regained interest. Wallet et $\mathrm{al}^{36}$ wanted to assess whether there was a significant relationship between recruited lung volume and change in density on digitally processed chest x-ray measured at 2 different levels of inspiratory plateau pressure corresponding to 2 PEEP levels in patients with acute lung injury or ARDS. Interestingly, digital chest $\mathrm{x}$-ray done at the bedside in acute lung injury/ARDS subjects was able to detect a reduction in density in preselected regions of interest between PEEP 5 and $15 \mathrm{~cm} \mathrm{H}_{2} \mathrm{O}$, which correlated with recruited lung volume. Compared to other methods of detecting recruitment, bedside digital chest radiography is readily available and does not require patient transport. However, the clinical usefulness remains to be evaluated.

Changes in lung volume can be evaluated by measuring variations in lung impedance using electrical impedance tomography. Riera et $\mathrm{al}^{37}$ used electrical impedance tomography to investigate the effects of high-flow nasal cannula and body position on global and regional endexpiratory lung impedance variation in healthy subjects. High-flow nasal cannula increased global end-expiratory lung impedance variation regardless of body position, suggesting an increase in functional residual capacity. Prone positioning was related to a more homogeneous distribution of end-expiratory lung impedance variation, whereas in supine position, end-expiratory lung impedance variation was higher in the ventral lung regions. Whether these effects can be reproduced in patients with acute lung injury remains to be investigated.

Using electrical impedance tomography, Mauri et al ${ }^{38}$ investigated the topographic distribution of $\mathrm{V}_{\mathrm{T}}$ during higher PEEP and enhanced spontaneous breathing in patients with ARDS undergoing pressure support ventilation. They found that higher PEEP and lower support levels increased the fraction of tidal ventilation reaching dependent lung regions, yielding more homogeneous ventilation and, possibly, better ventilation/perfusion coupling. However, even during pressure support ventilation, patients with ARDS can generate high transpulmonary pressure by strong spontaneous breathing efforts, which, in severe cases, could worsen lung injury. Therefore, when spontaneous breathing is preserved during mechanical ventilation, transpulmonary pressure and $\mathrm{V}_{\mathrm{T}}$ should be strictly controlled to prevent further lung injury. ${ }^{39,40}$

\section{Central Drive and Respiratory Muscles}

The contribution of the central respiratory drive to respiratory failure in patients admitted to the ICU because of a primary neuromuscular disease (NMD) with acute respi- ratory failure and in patients admitted to the ICU because of different medical or surgical diseases who developed ICU-acquired weakness, which is associated with weaning failure, has not been fully established. Rialp et $\mathrm{al}^{41}$ assessed the contribution of central respiratory drive to hypercapnic respiratory failure in NMDs by comparing the $\mathrm{CO}_{2}$ response and the duration of weaning from mechanical ventilation between a group of patients with NMDs and a group of quadriplegic patients due to ICU-acquired weakness. Subjects with acute hypercapnic respiratory failure secondary to NMD had a reduced hypercapnic drive response compared to subjects with ICU-acquired weakness, and reduced hypercapnic respiratory drive was associated with prolonged weaning from mechanical ventilation.

Respiratory muscle dysfunction could develop in critically ill patients and contribute to prolonged weaning from mechanical ventilation. Because diaphragmatic abnormalities at the initial phase of critical illness remain poorly documented in humans, Demoule et al ${ }^{42}$ determined the incidence, risk factors, and prognostic impact of diaphragmatic impairment on ICU admission. In 85 patients, the diaphragm was assessed by twitch tracheal pressure in response to bilateral anterior magnetic phrenic nerve stimulation. They found that ICU survivors had higher twitch tracheal pressure on admission, and this was also true for hospital survivors versus non-survivors. In addition, diaphragm dysfunction on ICU admission was frequent and was related to sepsis and the severity of the ongoing disease. Finally, diaphragm dysfunction was associated with a poorer prognosis but not with a longer duration of mechanical ventilation. Therefore, identifying acute diaphragm failure on ICU admission could then have an impact on subsequent clinical management.

Several methods have been used to assess diaphragmatic performance and derived variables like work of breathing in critically ill patients. However, these methods are not routinely used, and there is a need for simple and accurate methods to be used permanently at bedside. Because ultrasonography-based determination of diaphragm excursions may help identify patients with diaphragmatic dysfunction during weaning from mechanical ventilation, Vivier et $\mathrm{al}^{43}$ evaluated diaphragm thickness variation measured in the zone of apposition in 12 patients requiring planned noninvasive ventilation after extubation while spontaneously breathing and during noninvasive ventilation at 3 levels of pressure support. Diaphragmatic pressure-time product per breath and the diaphragm thickening fraction at the zone of apposition decreased as the level of pressure support increased. Therefore, ultrasonographic assessment of the diaphragm is an easy and noninvasive method that may prove useful in evaluating diaphragmatic function and its contribution to respiratory work load in critically ill patients. 


\section{Information Technologies}

The data generated in the process of care of critically ill patients are underused, and most are simply wasted. This is due in part to the difficulty of accessing, processing, integrating, organizing, and using data from different sources (paper charts, electronic records, digital waveforms) and the notable variability in clinical documentation methods and different medical devices. In the absence of a practical way to systematically capture, analyze, and integrate the information, care of the critically ill has remained a process based on physiologic knowledge, empiricism, and experience. Initiatives should arise to help clinicians to construct dynamic learning systems, fully supported by engineers to collaborate on the daily translation of questions into strategies for database interrogation, modeling, and analysis. ${ }^{44}$

Pickering et $\mathrm{al}^{45}$ determined the clinical information needs of ICU physicians compared to the data available within an electronic medical record to facilitate the development of information systems that prioritize the presentation of high-value data and reduce information overload. The clinical information used by physicians during the initial diagnosis and treatment of admitted patients was captured using a questionnaire. Clinical information concepts were ranked according to the frequency of reported use (primary outcome) and were compared to information availability in the electronic medical record (secondary outcome). Interestingly, only 11 concepts were used by physicians per patient admission encounter, with four used $>50 \%$ of the time, and over $25 \%$ of the clinical data available in the electronic medical record were never used. Physicians use a limited number of clinical information concepts at the time of patient admission to the ICU. This fact is particularly dramatic in patients receiving mechanical ventilation.

Three very interesting reviews ${ }^{46-48}$ highlighting the importance of the interpretation of asynchronies between the patient and the mechanical ventilator were published during 2013. Patient-ventilator asynchronies are common, decrease comfort, prolong mechanical ventilation and ICU stay, and might lead to worse outcome. To date, the detection of asynchronies usually depends on health care staff observing ventilator waveforms, and many events go undetected. Technological complexity has made it impossible to continuously evaluate asynchronies between the patient and the ventilator throughout the course of mechanical ventilation. Through collaborative work between clinicians and engineers, various authors have pointed out the need for computerized systems to facilitate recognition of these events and to provide contextual information when professionals are not present at the bedside.

Blanch et $\mathrm{al}^{49}$ developed an algorithm to automatically diagnose ineffective expiratory efforts in expiratory flow- time waveforms. This approach analyzes the air-flow curve from a data set recorded continuously $24 \mathrm{~h}$ a day, is not limited to a specific device or ventilation mode, and is not influenced by the presence of secretions. Tested on a small sample of patients, the accuracy of the computerized automatic ineffective inspiratory efforts algorithm during expiration was similar to that when compared to expert critical care staff and the electrical activity of the diaphragm. Sinderby et $\mathrm{al}^{50}$ tested a new method of automatically detecting, quantifying, and displaying patient-ventilator interaction based on the measurements of electrical activity of the diaphragm and airway pressure waveforms. This method provides clinicians with a dashboard style of graphical display that allows a rapid overview of patient-ventilator interaction and breathing pattern.

\section{Invasive Mechanical Ventilation}

A number of interesting papers on invasive mechanical ventilation published in 2013 focused on the following themes: high-frequency oscillatory ventilation (HFOV) in adult patients with ARDS, ${ }^{51,52}$ the use of lung-protective ventilation outside the ICU, ${ }^{53}$ and tracheostomy timing and weaning. ${ }^{15,54}$

\section{High-Frequency Oscillatory Ventilation in Adult Patients With ARDS}

A number of preclinical and clinical studies have demonstrated that ventilator-induced lung injury, including repetitive overstretching (volutrauma) and collapse (atelectrauma) during tidal ventilation, can lead to a local and systemic inflammatory response that contributes to the development of multiple-organ failure and death in patients with ARDS. ${ }^{55}$ Indeed, subsequent randomized controlled trials (RCTs) evaluating these principles of lung-protective ventilation in ARDS support the use of smaller $\mathrm{V}_{\mathrm{T}}$ values ( 6 vs $12 \mathrm{~mL} / \mathrm{kg}$ predicted body weight $)^{28}$ and higher levels of PEEP. ${ }^{56}$ HFOV represents an alternative mode of ventilation that may be ideal for lung protection by delivering very small $\mathrm{V}_{\mathrm{T}}$ values $(1-4 \mathrm{~mL} / \mathrm{kg}$ predicted body weight) to minimize over-distention, with relatively high mean airway pressures to prevent atelectrauma. ${ }^{57}$ Several small clinical trials evaluating the efficacy of HFOV in patients with ARDS have suggested both improved oxygenation and survival with this strategy. ${ }^{58}$ The putative benefits of HFOV in ARDS were evaluated in two large multi-center RCTs that were completed and reported in 2013. ${ }^{51,52}$

The Oscillation for Acute Respiratory Distress Syndrome Treated Early (OSCILLATE) trial ${ }^{51}$ randomized patients with moderate/severe ARDS to a conventional mechanical ventilation strategy using recruitment maneuvers and higher levels of PEEP (adapted from the LOV study) ${ }^{33}$ or a HFOV 
strategy using high mean airway pressures and respiratory frequencies aimed at maximizing lung recruitment and using small $\mathrm{V}_{\mathrm{T}}$ values. Although originally planned to enroll 1,200 patients, on the recommendations of the Data Safety Monitoring Board, the trial was stopped early after 548 patients had been randomized. In-hospital mortality was significantly greater in the HFOV group (relative risk [RR] $1.33,95 \%$ CI $1.09-1.64)$. There was no subgroup of patients who seemed to benefit from HFOV, including those with greater baseline severity of ARDS (as measured by oxygenation and respiratory compliance). Patients randomized to HFOV received significantly greater doses of sedation (median 199 vs $141 \mathrm{mg}$ of midazolam/d, $P<$ $.001)$ and more neuromuscular blockers (83 vs $68 \%, P<$ .001 ) and required more vasoactive agents (91 vs $84 \%$, $P=.01)$.

The Oscillation in ARDS (OSCAR) trial ${ }^{52}$ randomized patients with moderate/severe ARDS requiring mechanical ventilation to HFOV or usual ventilatory care. As a pragmatic trial, patients in the usual care group were treated according to local practices in the participating ICUs. Importantly, patients in the usual care group received average $\mathrm{V}_{\mathrm{T}}$ values $>8 \mathrm{~mL} / \mathrm{kg}$ predicted body weight. There was no significant difference in crude 30 -day (42 vs $41 \%$, $P=.85$ ) or hospital (50 vs $48 \%, P=.62$ ) all-cause mortality between the HFOV and usual care groups. The results were unchanged after adjustment for a number of baseline covariates (OR for survival in the usual care group $1.03,95 \%$ CI .75-1.40). Similar to the OSCILLATE trial, patients in the HFOV group were exposed to more neuromuscular blockers and sedatives.

Given the results of these 2 RCTs, the routine use of HFOV in adult patients with moderate ARDS is not warranted. A number of potential mechanisms may have led to these disappointing results, including the deleterious effects of heavy sedation and neuromuscular blockade, hemodynamic compromise due to adverse effects of high mean airway pressure on the right ventricle, ${ }^{59}$ or increased ventilator-induced lung injury among HFOV non-responders. ${ }^{60}$ However, given that patients with refractory hypoxemia in the control group of the OSCILLATE trial could be rescued with HFOV (12\%), clinicians could consider HFOV in carefully selected patients with severe ARDS who were failing optimal conventional mechanical ventilation and, perhaps in the absence of contraindications, a trial of prone positioning. ${ }^{61}$ Patients should be assessed for their response to HFOV: if there is a significant increase in vasopressor requirements and/or failure to improve oxygenation, these patients should be switched back to conventional mechanical ventilation. Future clinical trials should consider individualized approaches to setting HFOV in patients with ARDS, including dynamic monitoring of lung recruitment and right ventricular function.

\section{Lung-Protective Ventilation Outside the ICU}

Although lung-protective ventilation with pressure and volume limitation has become the cornerstone of management in critically ill patients with ARDS, ${ }^{28}$ larger $\mathrm{V}_{\mathrm{T}}$ values $(10-15 \mathrm{~mL} / \mathrm{kg}$ predicted body weight) have been traditionally employed in anesthetized patients undergoing surgery. However, increasing evidence suggests that lungprotective ventilation may mitigate ventilator-induced lung injury in patients without ARDS and may result in fewer pulmonary complications in the postoperative setting. ${ }^{62} \mathrm{To}$ that end, the multi-center Intra-Operative Protective Ventilation (IMPROVE) trial $^{53}$ randomized 400 patients at intermediate to high risk of pulmonary complications after major abdominal surgery to either a lung-protective strategy (including $\mathrm{V}_{\mathrm{T}} 6-8 \mathrm{~mL} / \mathrm{kg}$ predicted body weight, PEEP $6-8 \mathrm{~cm} \mathrm{H}_{2} \mathrm{O}$, and recruitment maneuvers) or a non-protective strategy using $\mathrm{V}_{\mathrm{T}} \quad 10-12 \mathrm{~mL} / \mathrm{kg}$ predicted body weight without any PEEP or recruitment maneuvers. Plateau pressure was kept below $30 \mathrm{~cm} \mathrm{H}_{2} \mathrm{O}$ in both groups.

Patients in the lung-protective group had significantly fewer major pulmonary (eg, atelectasis, ARDS, need for mechanical ventilation) and extrapulmonary (eg, systemic inflammatory response syndrome, sepsis) complications occurring by day 7 (adjusted RR 0.40, 95\% CI $0.24-$ 0.68 ), their primary composite outcome, and day 30 (adjusted RR $0.45,95 \%$ CI $0.28-0.73$ ) after surgery. It is important to note that a greater incidence of atelectasis, pneumonia, and need for noninvasive ventilation in the non-protective groups were the main drivers for the observed difference between groups, as these complications may have a limited impact on patient-important outcomes. Indeed, there were no differences in 30-day mortality or ICU stay between groups, although patients in the lungprotective group had a shorter median hospital stay (adjusted RR -2.45 days, $95 \%$ CI -4.17 to -0.72 ).

This randomized trial demonstrated that the use of intraoperative lung-protective ventilation led to decreased postoperative complications and health care utilization after major abdominal surgery. This study is consistent with recent reports that extending the use of lung-protective ventilation outside of the ICU and patients with ARDS may have additional benefits. ${ }^{62}$ Although this study adds to the growing evidence suggesting that any patient receiving mechanical ventilation may benefit from a lungprotective strategy, more definitive studies are needed before widespread adoption occurs across all patient populations.

\section{Tracheostomy Timing and Weaning}

Among patients requiring PMV, placement of a tracheostomy may be associated with a number of benefits, including improved oral care and the opportunity for the 
patient to eat and speak, and may facilitate trials of unassisted breathing. The challenge is, however, determining who will need PMV and the optimal timing for tracheostomy. ${ }^{63} \mathrm{~A}$ systematic review and meta-analysis suggested that early tracheostomy may be associated with a shorter duration of mechanical ventilation and hospital stay, ${ }^{64}$ and at least one study has suggested a reduction in hospital mortality. ${ }^{65}$

These results were challenged by a multi-center RCT that evaluated early (6-9 days) versus late (13-15 days) tracheostomy in 419 patients after initiation of mechanical ventilation. ${ }^{66}$ There was no difference in the primary end point of VAP, and 39\% of patients had an adverse event associated with the placement of the tracheostomy. Importantly, nearly half of the patients randomized to the late group never required a tracheostomy, suggesting that clinicians are poor at determining who needs this procedure. ${ }^{67}$ As described previously in this review, the TracMan trial ${ }^{15}$ also demonstrated a lack of important benefits with early tracheostomy, as well as a similar proportion of patients who did not subsequently require a tracheostomy if clinicians waited long enough. This suggests that a waitand-see approach may obviate an unnecessary procedure in many patients. Until more robust tools are developed to aid clinicians in predicting who will require PMV,63,67 tracheostomy should be delayed until at least 10 days after the initiation of mechanical ventilation.

In the United States, many tracheostomized patients requiring PMV will eventually be transferred to a long-term acute care hospital (LTACH) that specializes in the care of these patients. ${ }^{68}$ However, despite the increasing use of these facilities, little is known about the optimal management of patients at these centers, particularly how to wean those requiring PMV. ${ }^{69}$ To that end, Jubran et al ${ }^{54}$ designed an RCT enrolling 500 patients requiring PMV ( $>21$ days) with a tracheostomy who were referred for weaning at a single LTACH. Interestingly, only 316 (63\%) patients failed the 5-day unassisted breathing screening period for the study and were ultimately randomized to one of two common weaning methods: gradual reduction in pressure support versus lengthening trials of unassisted breathing through a tracheostomy collar (TC).

There was no significant difference in the proportion of patients successfully weaned in the TC versus pressure support groups (53 vs $45 \%, P=.14$ ). However, median weaning time was shorter in the TC group (15 vs 19 days, $P=.004)$. After adjusting for baseline characteristics, the TC method was associated with significantly higher rates of successful weaning (hazard ratio 1.45 , 95\% CI 1.031.98). However, the relationship between timing of initial screening failure and weaning method on weaning duration was not constant over time. While the weaning method was not significantly associated with weaning duration among patients in the early-failure (0-12 h) group during the 5-day screening period, among patients in the latefailure $(12-120 \mathrm{~h})$ group, the weaning method was the only factor associated with weaning duration, with the rate of successful weaning being significantly greater with the TC method (hazard ratio 3.33, 95\% CI 1.44-7.77). Importantly, adverse events and long-term mortality (at 6 and $12 \mathrm{mo}$ ) were similar in the 2 groups.

Prior to this study, the most effective strategy for weaning patients requiring PMV at LTACHs was unknown, and this study provides data supporting the use of daily unassisted breathing trials in these patients. Perhaps even more striking is the fact that nearly one third of patients referred to an LTACH for failure to wean were able to breathe without assistance during the initial screening period, suggesting poor clinician recognition of patients who truly require ongoing ventilatory support or failure to attempt to wean. ${ }^{69}$ Finally, this clinical trial demonstrates that although research in an LTACH is difficult (this trial recruited patients over a 10-year period), it is feasible and can generate important data to help define the best practices in these settings.

\section{Summary}

In this paper, the important recent literature on airway management, noninvasive monitoring, and invasive mechanical ventilation is reviewed. It is our hope that this will help to familiarize the reader with the important literature in these subject areas.

\section{REFERENCES}

1. Adams MC, Schmidt U, Hess DR, Stelfox HT, Bittner EA. Examination of patterns in intubation by an emergency airway team at a large academic center: higher frequency during daytime hours. Respir Care. 2013 Oct 15. doi:10.4187/respcare.02432.

2. Joffe AM, Liew EC, Olivar H, Dagal AH, Grabinsky A, Hallman M, Treggiari MM. A national survey of airway management training in United States internal medicine-based critical care fellowship programs. Respir Care 2012;57(7):1084-1088.

3. Berg RA, Hemphill R, Abella BS, Aufderheide TP, Cave DM, Hazinski MF, et al. Part 5: adult basic life support: 2010 American Heart Association guidelines for cardiopulmonary resuscitation and emergency cardiovascular care. Circulation 2010;122 (18 Suppl 3):S685S705.

4. Pabst D, Römer S, Samol A, Kümpers P, Waltenberger J, Lebiedz P. Predictors and outcome of early-onset pneumonia after out-of-hospital cardiac arrest. Respir Care 2013;58(9):1514-1520.

5. Hagberg C, Bogomolny Y, Gilmore C, Gibson V, Kaitner M, Khurana $\mathrm{S}$. An evaluation of the insertion and function of a new supraglottic airway device, the King LT, during spontaneous ventilation. Anesth Analg 2006;102(2):621-625.

6. Khaja SF, Chang KE. Airway algorithm for the management of patients with a King LT. Laryngoscope. 2013 Sep 18. doi:10.1002/ lary. 24374

7. Gaither JB, Matheson J, Eberhardt A, Colwell CB. Tongue engorgement associated with prolonged use of the King-LT laryngeal tube device. Ann Emerg Med 2010;55(4):367-369. 


\section{Respiratory CARE Year In Review 2013}

8. Ostermayer DG, Gausche-Hill M. Supraglottic airways: the history and current state of prehospital airway adjuncts. Prehosp Emerg Care 2014;18(1):106-115.

9. Hasegawa K, Hagiwara Y, Imamura T, Chiba T, Watase H, Brown CA, Brown DF. Increased incidence of hypotension in elderly patients who underwent emergency airway management: an analysis of a multi-centre prospective observational study. Int J Emerg Med 2013;6(1):12.

10. Heffner AC, Swords DS, Neale MN, Jones AE. Incidence and factors associated with cardiac arrest complicating emergency airway management. Resuscitation 2013;84(11):1500-1504.

11. Law JA, Broemling N, Cooper RM, Drolet P, Duggan LV, Griesdale $\mathrm{DE}$, et al. The difficult airway with recommendations for management-part 1-difficult tracheal intubation encountered in an unconscious/induced patient. Can J Anaesth 2013;60(11):1089-1118.

12. Law JA, Broemling N, Cooper RM, Drolet P, Duggan LV, Griesdale $\mathrm{DE}$, et al. The difficult airway with recommendations for management-part 2-the anticipated difficult airway. Can J Anaesth 2013; 60(11):1119-1138.

13. Shetty K, Nayyar V, Stachowski E, Byth K. Training for cricothyroidotomy. Anaesth Intensive Care 2013;41(5):623-630.

14. Hamaekers AE, Henderson JJ. Equipment and strategies for emergency tracheal access in the adult patient. Anaesthesia 2011;66(Suppl 2):65-80.

15. Young D, Harrison DA, Cuthbertson BH, Rowan K, TracMan Collaborators. Effect of early vs late tracheostomy placement on survival in patients receiving mechanical ventilation: the TracMan randomized trial. JAMA 2013;309(20):2121-2129.

16. Fisher DF, Kondili D, Williams J, Hess DR, Bittner EA, Schmidt UH. Tracheostomy tube change before day 7 is associated with earlier use of speaking valve and earlier oral intake. Respir Care 2013;58(2):257-263.

17. Ledgerwood LG, Salgado MD, Black H, Yoneda K, Sievers A, Belafsky PC. Tracheotomy tubes with suction above the cuff reduce the rate of ventilator-associated pneumonia in intensive care unit patients. Ann Otol Rhinol Laryngol 2013;122(1):3-8.

18. Chou HC, Chong KM, Sim SS, Ma MH, Liu SH, Chen NC, et al. Real-time tracheal ultrasonography for confirmation of endotracheal tube placement during cardiopulmonary resuscitation. Resuscitation 2013;84(12):1708-1712.

19. Wang ML, Schuster KM, Bhattacharya B, Maung AA, Kaplan LJ, Davis KA. Repositioning endotracheal tubes in the intensive care unit: depth changes poorly correlate with postrepositioning radiographic location. J Trauma Acute Care Surg 2013;75(1):146-149.

20. De Jong A, Molinari N, Terzi N, Mongardon N, Arnal JM, Guitton $\mathrm{C}$, et al. Early identification of patients at risk for difficult intubation in the intensive care unit: development and validation of the MACOCHA score in a multicenter cohort study. Am J Respir Crit Care Med 2013;187(8):832-839.

21. Huang CT, Yu CJ. Conventional weaning parameters do not predict extubation outcome in intubated subjects requiring prolonged mechanical ventilation. Respir Care 2013;58(8):1307-1314.

22. Weaver LK, Churchill SK, Deru K, Cooney D. False positive rate of carbon monoxide saturation by pulse oximetry of emergency department patients. Respir Care 2013;58(2):232-240.

23. Sebbane M, Claret PG, Mercier G, Lefebvre S, Théry R, Dumont R, et al. Emergency department management of suspected carbon monoxide poisoning: role of pulse CO-oximetry. Respir Care 2013;58(10): 1614-1620.

24. Blanch L, Maspons R, Palomar G. Do we need to innovate in critical care practice? Crit Care 2013;17(4):166.

25. Siobal MS, Ong H, Valdes J, Tang J. Calculation of physiologic dead space: comparison of ventilator volumetric capnography to measure- ments by metabolic analyzer and volumetric $\mathrm{CO}_{2}$ monitor. Respir Care 2013;58(7):1143-1151.

26. Pintado MC, de Pablo R, Trascasa M, Milicua JM, Rogero S, Daguerre M, et al. Individualized PEEP setting in subjects with ARDS: a randomized controlled pilot study. Respir Care 2013;58(9):14161423.

27. Suter PM, Fairley HB, Isenberg MD. Effect of tidal volume and positive end-expiratory pressure on compliance during mechanical ventilation. Chest 1978;73(2):158-162.

28. The Acute Respiratory Distress Syndrome Network. Ventilation with lower tidal volumes as compared with traditional tidal volumes for acute lung injury and the acute respiratory distress syndrome. N Engl J Med 2000;342(18):1301-1308.

29. Chiumello D, Cressoni M, Carlesso E, Caspani ML, Marino A, Gallazzi E, et al. Bedside selection of positive end-expiratory pressure in mild, moderate, and severe acute respiratory distress syndrome. Crit Care Med 2014;42(2):252-264.

30. Grasso S, Terragni P, Mascia L, Fanelli V, Quintel M, Herrmann P, et al. Airway pressure-time curve profile (stress index) detects tidal recruitment/hyperinflation in experimental acute lung injury. Crit Care Med 2004;32(4):1018-1027.

31. Mercat A, Richard JC, Vielle B, Jaber S, Osman D, Diehl JL, et al. Positive end-expiratory pressure setting in adults with acute lung injury and acute respiratory distress syndrome: a randomized controlled trial. JAMA 2008;299(6):646-655.

32. Talmor D, Sarge T, Malhotra A, O'Donnell CR, Ritz R, Lisbon A, et al. Mechanical ventilation guided by esophageal pressure in acute lung injury. N Engl J Med 2008;359(20):2095-2104.

33. Meade MO, Cook DJ, Guyatt GH, Slutsky AS, Arabi YM, Cooper DJ, et al. Ventilation strategy using low tidal volumes, recruitment maneuvers, and high positive end-expiratory pressure for acute lung injury and acute respiratory distress syndrome: a randomized controlled trial. JAMA 2008;299(6):637-645.

34. ARDS Definition Task Force, Ranieri VM, Rubenfeld GD, Thompson BT, Ferguson ND, Caldwell E, et al. Acute respiratory distress syndrome: the Berlin Definition. JAMA 2012;307(23):2526-2533.

35. Protti A, Andreis DT, Monti M, Santini A, Sparacino CC, Langer T, et al. Lung stress and strain during mechanical ventilation: any difference between statics and dynamics? Crit Care Med 2013;41(4): 1046-1055.

36. Wallet F, Delannoy B, Haquin A, Debord S, Leray V, Bourdin G, et al. Evaluation of recruited lung volume at inspiratory plateau pressure with PEEP using bedside digital chest $\mathrm{x}$-ray in patients with acute lung injury/ARDS. Respir Care 2013;58(3):416-423.

37. Riera J, Pérez P, Cortés J, Roca O, Masclans JR, Rello J. Effect of high-flow nasal cannula and body position on end-expiratory lung volume: a cohort study using electrical impedance tomography. Respir Care 2013;58(4):589-596.

38. Mauri T, Bellani G, Confalonieri A, Tagliabue P, Turella M, Coppadoro A, et al. Topographic distribution of tidal ventilation in acute respiratory distress syndrome: effects of positive end-expiratory pressure and pressure support. Crit Care Med 2013;41(7):1664-1673.

39. Yoshida T, Uchiyama A, Matsuura N, Mashimo T, Fujino Y. The comparison of spontaneous breathing and muscle paralysis in two different severities of experimental lung injury. Crit Care Med 2013; 41(2):536-545

40. L'Her E, Deye N, Lellouche F, Taille S, Demoule A, Fraticelli A, et al. Physiologic effects of noninvasive ventilation during acute lung injury. Am J Respir Crit Care Med 2005;172(9):1112-1118.

41. 22. Rialp G, Raurich JM, Llompart-Pou JA, Ayestarán I, Ibáñez J. Central respiratory drive in patients with neuromuscular diseases. Respir Care 2013;58(3):450-457.

42. Demoule A, Jung B, Prodanovic H, Molinari N, Chanques G, Coirault $\mathrm{C}$, et al. Diaphragm dysfunction on admission to the intensive 


\section{Respiratory Care Year In Review 2013}

care unit. Prevalence, risk factors, and prognostic impact-a prospective study. Am J Respir Crit Care Med 2013;188(2):213-219.

43. Vivier E, Mekontso Dessap A, Dimassi S, Vargas F, Lyazidi A, et al. Diaphragm ultrasonography to estimate the work of breathing during non-invasive ventilation. Intensive Care Med 2012;38(5):796-803.

44. Celi LA, Mark RG, Stone DJ, Montgomery RA. "Big data" in the intensive care unit. Closing the data loop. Am J Respir Crit Care Med 2013;187(11):1157-1160.

45. Pickering BW, Gajic O, Ahmed A, Herasevich V, Keegan MT. Data utilization for medical decision making at the time of patient admission to ICU. Crit Care Med 2013;41(6):1502-1510.

46. Murias G, Villagra A, Blanch L. Patient-ventilator dyssynchrony during assisted invasive mechanical ventilation. Minerva Anestesiol 2013;79(4):434-444.

47. Gilstrap D, MacIntyre N. Patient-ventilator interactions. Implications for clinical management. Am J Respir Crit Care Med 2013; 188(9):1058-1068.

48. Branson RD, Blakeman TC, Robinson BR. Asynchrony and dyspnea. Respir Care 2013;58(6):973-989.

49. Blanch L, Sales B, Montanya J, Lucangelo U, Garcia-Esquirol O, Villagra A, et al. Validation of the Better Care ${ }^{\circledR}$ system to detect ineffective efforts during expiration in mechanically ventilated patients: a pilot study. Intensive Care Med 2012;38(5):772-780.

50. Sinderby C, Liu S, Colombo D, Camarotta G, Slutsky AS, Navalesi $\mathrm{P}$, Beck J. An automated and standardized neural index to quantify patient-ventilator interaction. Crit Care 2013;17(5):R239.

51. Ferguson ND, Cook DJ, Guyatt GH, Mehta S, Hand L, Austin P, et al. High-frequency oscillation in early acute respiratory distress syndrome. N Engl J Med 2013;368(9):795-805.

52. Young D, Lamb SE, Shah S, MacKenzie I, Tunnicliffe W, Lall R, et al. High-frequency oscillation for acute respiratory distress syndrome. N Engl J Med 2013;368(9):806-813.

53. Futier E, Constantin JM, Paugam-Burtz C, Pascal J, Eurin M, Neuschwander A, et al. A trial of intraoperative low-tidal-volume ventilation in abdominal surgery. N Engl J Med 2013;369(5):428437.

54. Jubran A, Grant BJ, Duffner LA, Collins EG, Lanuza DM, Hoffman LA, et al. Effect of pressure support vs unassisted breathing through a tracheostomy collar on weaning duration in patients requiring prolonged mechanical ventilation: a randomized trial. JAMA 2013; 309(7):671-677.

55. Slutsky AS, Ranieri VM. Ventilator-induced lung injury. N Engl J Med 2013;369(22):2126-2136.

56. Briel M, Meade M, Mercat A, Brower RG, Talmor D, Walter SD, et al. Higher vs lower positive end-expiratory pressure in patients with acute lung injury and acute respiratory distress syndrome: systematic review and meta-analysis. JAMA 2010;303(9):865-873.

57. Fan E, Stewart TE. New modalities of mechanical ventilation: highfrequency oscillatory ventilation and airway pressure release ventilation. Clin Chest Med 2006;27(4):615-625

58. Sud S, Sud M, Friedrich JO, Meade MO, Ferguson ND, Wunsch H, Adhikari NK. High frequency oscillation in patients with acute lung injury and acute respiratory distress syndrome (ARDS): systematic review and meta-analysis. BMJ 2010;340:c2327.

59. Guervilly C, Forel JM, Hraiech S, Demory D, Allardet-Servent J, Adda $\mathrm{M}$, et al. Right ventricular function during high-frequency oscillatory ventilation in adults with acute respiratory distress syndrome. Crit Care Med 2012;40(5):1539-1545.

60. Goligher EC, Ferguson ND. Re-evaluating high-frequency oscillation for ARDS: would a targeted approach be successful? Crit Care 2013;17(2):133.

61. Guérin C, Reignier J, Richard JC, Beuret P, Gacouin A, Boulain T, et al. Prone positioning in severe acute respiratory distress syndrome. N Engl J Med 2013;368(23):2159-2168.

62. Serpa Neto A, Cardoso SO, Manetta JA, Pereira VG, Espósito DC, Pasqualucci Mde O, et al. Association between use of lung-protective ventilation with lower tidal volumes and clinical outcomes among patients without acute respiratory distress syndrome: a meta-analysis. JAMA 2012;308(16):1651-1659.

63. Angus DC. When should a mechanically ventilated patient undergo tracheostomy? JAMA 2013;309(20):2163-2164.

64. Griffiths J, Barber VS, Morgan L, Young JD. Systematic review and meta-analysis of studies of the timing of tracheostomy in adult patients undergoing artificial ventilation. BMJ 2005;330(7502):1243.

65. Rumbak MJ, Newton M, Truncale T, Schwartz SW, Adams JW, Hazard PB. A prospective, randomized, study comparing early percutaneous dilational tracheotomy to prolonged translaryngeal intubation (delayed tracheotomy) in critically ill medical patients. Crit Care Med 2004;32(8):1689-1694

66. Terragni PP, Antonelli M, Fumagalli R, Faggiano C, Berardino M, Pallavicini FB, et al. Early vs late tracheotomy for prevention of pneumonia in mechanically ventilated adult ICU patients: a randomized controlled trial. JAMA 2010;303(15):1483-1489.

67. Scales DC, Ferguson ND. Early vs late tracheotomy in ICU patients. JAMA 2010;303(15):1537-1538.

68. Kahn JM, Benson NM, Appleby D, Carson SS, Iwashyna TJ. Longterm acute care hospital utilization after critical illness. JAMA 2010; 303(22):2253-2259.

69. Kahn JM, Carson SS. Generating evidence on best practice in longterm acute care hospitals. JAMA 2013;309(7):719-720. 